\title{
TITLE:
}

\section{PROMINENCE ACTIVATION BY CORONAL FAST MODE SHOCK}

AUTHOR(S):

Takahashi, Takuya; Asai, Ayumi; Shibata, Kazunari

\section{CITATION:}

Takahashi, Takuya ...[et al]. PROMINENCE ACTIVATION BY CORONAL FAST MODE SHOCK. The Astrophysical Journal 2015, 801(1): 37.

\section{ISSUE DATE:}

2015-02-27

URL:

http://hdl.handle.net/2433/198606

RIGHT:

(C) 2015. The American Astronomical Society. 


\title{
PROMINENCE ACTIVATION BY CORONAL FAST MODE SHOCK
}

\author{
Takuya Takahashi ${ }^{1,3}$, Ayumi Asai ${ }^{2}$, and Kazunari Shibata ${ }^{3}$ \\ 1 Department of Astronomy, Kyoto University, Sakyo, Kyoto, 606-8502, Japan; takahashi@kwasan.kyoto-u.ac.jp \\ 2 Unit of Synergetic Studies for Space, Kyoto University, Yamashina, Kyoto 607-8471, Japan \\ 3 Kwasan and Hida Observatories, Kyoto University, Yamashina, Kyoto 607-8471, Japan \\ Received 2014 September 6; accepted 2014 December 28; published 2015 February 27
}

\begin{abstract}
An X5.4 class flare occurred in active region NOAA11429 on 2012 March 7. The flare was associated with a very fast coronal mass ejection (CME) with a velocity of over $2500 \mathrm{~km} \mathrm{~s}^{-1}$. In the images taken with the Solar Terrestrial Relations Observatory-B/COR1, a dome-like disturbance was seen to detach from an expanding CME bubble and propagated further. A Type-II radio burst was also observed at the same time. On the other hand, in extreme ultraviolet images obtained by the Solar Dynamic Observatory/Atmospheric Imaging Assembly (AIA), the expanding dome-like structure and its footprint propagating to the north were observed. The footprint propagated with an average speed of about $670 \mathrm{~km} \mathrm{~s}^{-1}$ and hit a prominence located at the north pole and activated it. During the activation, the prominence was strongly brightened. On the basis of some observational evidence, we concluded that the footprint in AIA images and the ones in COR1 images are the same, that is, the MHD fast mode shock front. With the help of a linear theory, the fast mode Mach number of the coronal shock is estimated to be between 1.11 and 1.29 using the initial velocity of the activated prominence. Also, the plasma compression ratio of the shock is enhanced to be between 1.18 and 2.11 in the prominence material, which we consider to be the reason for the strong brightening of the activated prominence. The applicability of linear theory to the shock problem is tested with a nonlinear MHD simulation.
\end{abstract}

Key words: magnetohydrodynamics (MHD) - shock waves - Sun: corona - Sun: filaments, prominences - Sun: flares

\section{INTRODUCTION}

The solar corona is a place where plasma transient phenomena such as flares, coronal mass ejections (CMEs), and jets are continuously observed. These are vigorously investigated in the framework of magnetohydrodynamics (e.g., Shibata \& Magara 2011). When magnetic energy is locally liberated in a short timescale as in flares or jets, magnetohydrodynamic shock waves are generated. The shock waves sometimes propagate into the interplanetary space and reach the Earth. The arrival of the shock waves to the Earth sometimes causes a sudden increase in the radiation level and a sudden change in the Earth's magnetic field by compressing it.

The existence of coronal disturbances associated with flares are suggested by some observation of far-away filament oscillation after flares. This is called the "winking filament" after the observational feature where the filament appeared and disappeared in $\mathrm{H} \alpha$ (Smith \& Harvey 1971). Sometimes, Type II solar radio bursts are observed associated with solar flares. Type II radio bursts are observed in dynamic radio spectra as bands slowly drifting down often in pairs differing in frequency by a factor of about two, and interpreted as the manifestation of coronal shock propagation (Wild et al. 1954; Kai 1970).

Globally propagating disturbances associated with solar flares are first observed in $\mathrm{H} \alpha$. Fan-shaped "wave fronts" were observed to propagate in the chromosphere after the occurrence of a flare (Moreton 1960). This phenomenon was named the "Moreton wave" after the name of the discoverer. The wave fronts of Moreton waves appear bright in the line center and blue wing and dark in the red wing followed by a fainter front whose signature of the intensity change is reversed (dark in the blue wing and bright in the red wing). This observational feature suggests a down-up swing of chromospheric plasma during the passage of the Moreton wave. The Moreton wave propagated with speeds between 500 and $1500 \mathrm{~km} \mathrm{~s}^{-1}$, and lasted for about 10 minutes. In some events, the Moreton wave front propagated faster than $2000 \mathrm{~km} \mathrm{~s}^{-1}$. The sound velocity in the chromosphere is about $10 \mathrm{~km} \mathrm{~s}^{-1}$. Fast mode magnetosonic wave speed is also of the same order. If Moreton waves are magnetohydrodynamic waves propagating in the chromosphere, they have to be strong shock waves with Mach numbers of over 10. It was difficult to accept that such strong shock waves propagate a long distance without any significant dissipation.

Uchida (1968) gave a comprehensive explanation of Moreton waves as a cross section between the coronal MHD fast mode shock front and the chromosphere. This model of Moreton waves was widely accepted as "Uchida's Moreton wave model" soon. According to the Uchida model, there has to be a coronal counterpart with the Moreton wave (namely the coronal MHD fast mode shock front). People call the expected coronal counterpart of the Moreton wave the "invisible" Moreton wave. Winking filaments were discussed as a result of the passage of invisible Moreton waves (Smith \& Harvey 1971; Eto et al. 2002; Okamoto et al. 2004).

In the 1990s, wave-like disturbances propagating in the corona after flares were observed with the Extreme Ultra Violet (EUV) Imaging Telescope (EIT; Delaboudinieře et al. 1995 ) on board the Solar and Heliospheric Observatory (SOHO; Domingo et al. 1995). This wave-like phenomenon was called the "EIT wave" after the name of the observational instrument. The EIT wave front propagated concentrically from the flare site with the speed of about $250 \mathrm{~km} \mathrm{~s}^{-1}$ (Thompson et al. 1999, 2000). At first, EIT waves were thought to be 
the expected coronal counterparts of Moreton waves. However, EIT waves and Moreton waves showed quite different observational features (Eto et al. 2002; Shibata et al. 2011; Zhang et al. 2011), which lead to a long standing discussion of the physical nature of EIT waves (Warmuth et al. 2001; Vršnak et al. 2002; Tripathi \& Raouafi 2007; Warmuth 2007; WillsDavey \& Attrill 2009; Gallagher \& Long 2010).

Recently, with the Atmospheric Imaging Assembly (AIA; Title \& AIA team 2006; Lemen et al. 2012) on board the Solar Dynamic Observatory (SDO; Pesnell et al. 2012), fast coronal waves have been observed associated with flares (e.g., Liu et al. 2010; Ma et al. 2011). Chen \& Wu (2011) found two different coexisting coronal waves, a slow coronal wave (i.e., EIT wave) and a fast coronal wave. These coronal disturbances are generally called EUV waves. Asai \& Ishii (2011) reported the first simultaneous observation of an $\mathrm{H} \alpha$ Moreton wave, a fast EUV wave and filament/prominence oscillations. In that paper, they showed that the fast EUV wave was the true coronal counterpart of the $\mathrm{H} \alpha$ Moreton wave. They also reported that after the Moreton wave disappeared, the fast EUV wave propagated further and activated a large amplitude filament/prominence oscillation.

Large amplitude prominence oscillations caused by EUV waves are often observed by SDO/AIA. Such prominence oscillations are generally driven in a very short timescale and behave as damping oscillators, while in some cases, the oscillations lead to the eruption of the prominences. They are sometimes driven by a sudden change of magnetic field structure through magnetic reconnections (Vršnak et al. 2007). These oscillations have been investigated in the context of diagnosis of physical quantities and the eruptive instability of the prominences (Isobe et al. 2007; Tripathi et al. 2009). The prominence activations by coronal shock waves and their subsequent oscillation provide us with an insight into physical properties of both coronal shock waves and the prominences (such as the width of the wave front and magnetic field structure supporting the prominences; Gilbert et al. 2008; Liu et al. 2013).

In this study, we analyzed the prominence activation process during the collision with an EUV wave associated with an X5.4 class flare on 2012 March 7. The unprecedented time and spatial resolution of multi-wavelength EUV data taken with SDO/AIA enable us to follow the prominence activation process in detail. We concluded, based on some observational evidence, that the EUV wave that activated the prominence is the same as the coronal shock wave propagating ahead of the CME flank, which was seen in the images taken with the inner Coronagraph (COR1) of the Sun Earth Connection Coronal and Heliospheric Investigation (SECCHI) on board the Solar Terrestrial Relations Observatory (STEREO; Kaiser et al. 2008; Driesman et al. 2008)-Behind satellite. We could explain the compression and acceleration of activated prominence as a result of coronal shock wave transmission into the dense prominence. Furthermore, we estimated the coronal shock strength using the initial velocity of the activated prominence.

In Section 2, we overview the observation of X5.4 class flare and associated coronal disturbances. In Section 3, we estimate the coronal shock strength using the initial velocity of activated prominence.

\section{OBSERVATION AND ANALYSIS}

\subsection{Overview of the Flare and CME}

Figure 1 shows the soft X-ray light curve taken with GOES satellite. The first X5.4 class flare occurred in active region (AR) NOAA11429 at 00:02UT and peaked at 00:24UT on 2012 March 7. One hour after the onset of the first flare, the second X1.3 class flare occurred at a different part of the same AR. The two flares each were accompanied by fast CMEs. The velocity of each CME is reported to be 2684 and $1825 \mathrm{~km} \mathrm{~s}^{-1}$, respectively. ${ }^{4}$ The abrupt change of the horizontal magnetic field and perpendicular Lorentz force near the polarity inversion line during X5.4 class flare are reported, and relationship between the abrupt change and the fast propagation velocity of the CME is pointed out (Petrie 2012; Wang et al. 2012). In this study, we investigate the coronal disturbances associated with the first flare of the two.

\subsection{A Disturbance Ahead of the CME Flank and the Type II Radio Burst}

On March 7, the AR NOAA11429 was located at (N17, E16) in the heliographic coordinate. The STEREO-B satellite was then observing the Sun at 118 degrees east of the Earth having captured the CME from the side. We analyzed the five minutes cadence data taken with STEREO-B/SECCHI/COR1.

Figure 2 shows the images taken with COR1 and EUVI on board the STEREO-B satellite. Figure 2(a) is the image taken with the extreme ultraviolet imager (EUVI; Wülser et al. 2004) $304 \AA$ pass band. In the EUVI $304 \AA$ image, we can see the polar prominence clearly. Figures $2(\mathrm{~b})-(\mathrm{d})$ are the difference images of the COR1 image with an embedded image of Figure 2(a). In Figure 2(b), a disturbance propagating ahead of the expanding CME plasma appeared at 00:25UT. This disturbance reached the sky just over the polar prominence at 00:31UT and propagated further as shown in (c) and (d). The average speed of propagation of the disturbance from 00:25UT to $00: 36 \mathrm{UT}$ was $420 \mathrm{~km} \mathrm{~s}^{-1}$ measured along the limb of the occulting disk of the COR1 images.

On the other hand, in the dynamic spectrum in the range between 25 and $2500 \mathrm{MHz}$, taken with the Hiraiso Radio Spectrograph (HIRAS, Kondo et al. 1995), the Type II radio burst was observed. The second harmonic component of the Type II radio burst appeared at 00:19:12UT in $200 \mathrm{MHz}$ and disappeared at 00:30:36UT in $50 \mathrm{MHz}$. From the average drift rate of the Type II radio burst, we can estimate the radial component of propagation velocity of the shock front ahead of the CME to be $672 \mathrm{~km} \mathrm{~s}^{-1}$ (Newkirk 1961; Mann et al. 1999). Because of the coincidence, we regarded the coronal disturbance ahead of the CME bubble in the COR 1 images to be the footprint of the shock wave that caused the Type II radio burst.

\subsection{EUV Observation}

In order to investigate the time evolution of the coronal disturbances, we used EUV images taken with SDO/AIA. In this study, we used two pass bands of AIA, namely 193 and $304 \AA$. The time cadence of AIA data is $12 \mathrm{~s}$ while we used $24 \mathrm{~s}$ cadence data in our analysis.

\footnotetext{
4 See http://cdaw.gsfc.nasa.gov/CME_list/UNIVERSAL/2012_03/univ2012_ 03.html.
} 


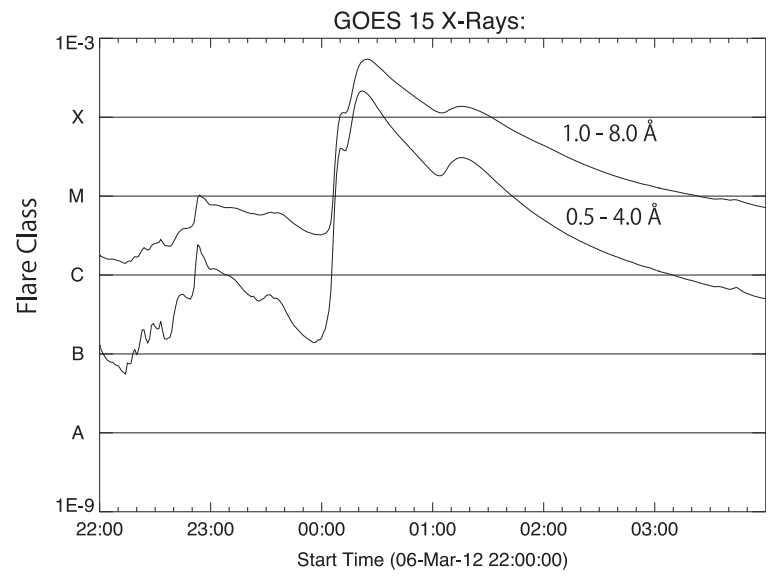

Figure 1. GOES Soft X-ray light curve. The X5.4 class flare occurred at 00:04UT and peaked at 00:24UT. One hour later the X1.3 flare occurred at a different part of the same active region.

Figure 3 shows the flare associated disturbances in the $193 \AA$ images. At 00:18 UT, dome-like structure appeared over the flaring AR. This dome-like structure kept on expanding and propagated upward from the solar disk. On the other hand, a strong disturbance appeared to the northeast of the AR at 00:23 UT, propagated in the lower corona toward the north pole, and hit a polar prominence located there. By comparing COR1 and AIA data, we concluded that the dome-like structure seen in AIA $193 \AA$ images is identical to the shock front ahead of CME seen in COR1 images. We consider the footprint propagated in the lower corona in AIA $193 \AA$ images to be the footprint of the shock (Figure 4).

These disturbances seen in EUV are both called "EUV waves." In the following, we distinguish the two disturbances as "dome-like structure" and "footprint" respectively. The dome-like structure expanded further and left away from the AIA field of view at 00:27UT. The footprint propagated further and hit the polar prominence at 00:33UT and caused large amplitude prominence oscillation. The average propagation velocity of the footprint between 00:18UT and 00:33UT was $670 \mathrm{~km} \mathrm{~s}^{-1}$. We can also see that the wave front of the footprint more and more inclined to the propagation direction during its propagation.

In Figure 3(b), the dome-like structure seems to already reach the polar prominence at 00:23UT. However, by comparing it with the shock front seen in STEREO-B/COR1 images, we notice that this is just because of a projection effect of three dimensionally expanding domes.

\subsection{Prominence Activation}

Taking a close look at the AIA $304 \AA$ images, we can clearly see how the large amplitude prominence oscillation was activated. Before the footprint "hit" the prominence at 00:33UT, the prominence was slowly swaying (Figure 5(a)). At 00:33 UT, the footprint of the coronal shock wave hits the prominence (Figure 5(b)). When it hit the edge of the prominence, the prominence was strongly brightened. The brightness was about three times that of the part before hit by the disturbance. After that the bright part propagated to the right in the image. The prominence was brightened from 00:33 UT to 00:37 UT, and during this time, the prominence material was accelerated from east to west in series (Figure 5(c)).
We can also see that the prominence was accelerated perpendicular to the wave front in AIA $193 \AA$ Amages. In $193 \AA$ images, the prominence was seen as a dark prominence (Figures 6(a)-(d)). It was brightened just when the footprint arrived but neither a long nor strong brightening could be seen as in $304 \AA$ images. From the AIA $193 \AA$ images, the propagation velocity of the wave front of the footprint just before the injection to the prominence was $380 \mathrm{~km} \mathrm{~s}^{-1}$ and the initial velocity of the activated prominence was $48 \mathrm{~km} \mathrm{~s}^{-1}$.

\section{ESTIMATION OF SHOCK STRENGTH IN THE CORONA}

We concluded that the footprint that activated the polar prominence was the shock wave that propagated ahead of the CME seen in the $S T E R E O-B / C O R 1$ images. Following are the reasons.

First, the propagation location of the footprint agreed well with the location of the shock wave ahead of the CME bubble. The shock wave ahead of the CME appeared in the STEREO-B/ COR1 image at 00:26 UT while the footprint in AIA $193 \AA$ images appeared at 00:23 UT. The timing of its appearance agreed well within the time cadence of STEREO-B/COR1. The shock wave in COR1 images passed the sky just over the polar prominence at 00:31 UT while the footprint in AIA $193 \AA$ images hit the prominence at 00:33 UT. We think that the two minute delay is due to the inclination of the wave front toward the propagation direction in the corona.

Second, the wave front of the footprint is inclined toward the propagation direction and the inclination increases with time. We believe this is because of wave refraction toward the photosphere because the phase velocity of the MHD fast mode wave would increase with height in the lower corona (Gopalswamy et al. 2001). The time evolution of the wave front agrees very well with the prediction of (Uchida 1968). Because of the gravitational stratification, the plasma density of the lower corona is higher, which made the footprint of the dome-like coronal shock front bright in EUV pass band.

Third, the polar prominence was strongly brightened and, at the same time, accelerated perpendicularly to the wave front. This behavior can be explained simply as a result of the injection of a compressive wave into the prominence.

Looking at the prominence activation in the direction perpendicular to the wave front, we can regard this process as one-dimensional fast mode shock wave transmission from corona into the dense prominence. In the linear case, the continuity of energy flux and momentum flux of the wave are written as

$$
\begin{gathered}
\rho_{c}\left(V_{i}^{2}-V_{r}^{2}\right) C_{c}=\rho_{p} V_{t}^{2} C_{p} \\
\rho_{c}\left(V_{i}-V_{r}\right) C_{c}=\rho_{p} V_{t} C_{p},
\end{gathered}
$$

which reduces the equation

$$
V_{i}=\frac{1}{2}\left(1+\frac{\rho_{p} C_{p}}{\rho_{c} C_{c}}\right) V_{t},
$$

where $\rho, V$, and $C$ are plasma density, velocity amplitude of the wave, and phase velocity of the MHD fast mode wave, respectively. The subscripts $i, t$, and $r$ of $V$ denote injected, transmitted, and reflected waves, respectively, and the subscripts $c$ and $p$ denote the quantities in the corona and prominence, respectively. 

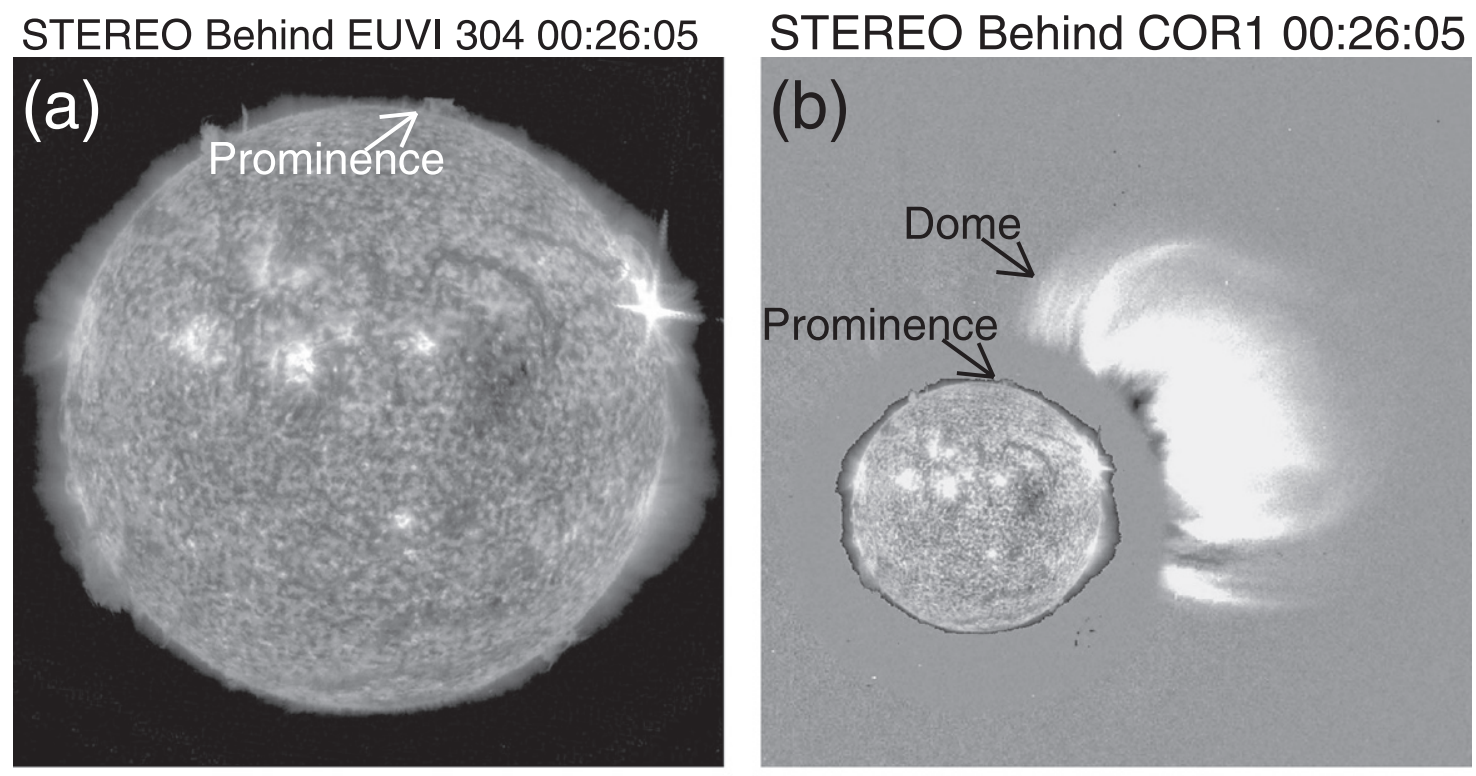

\section{STEREO Behind COR1 00:31:05}
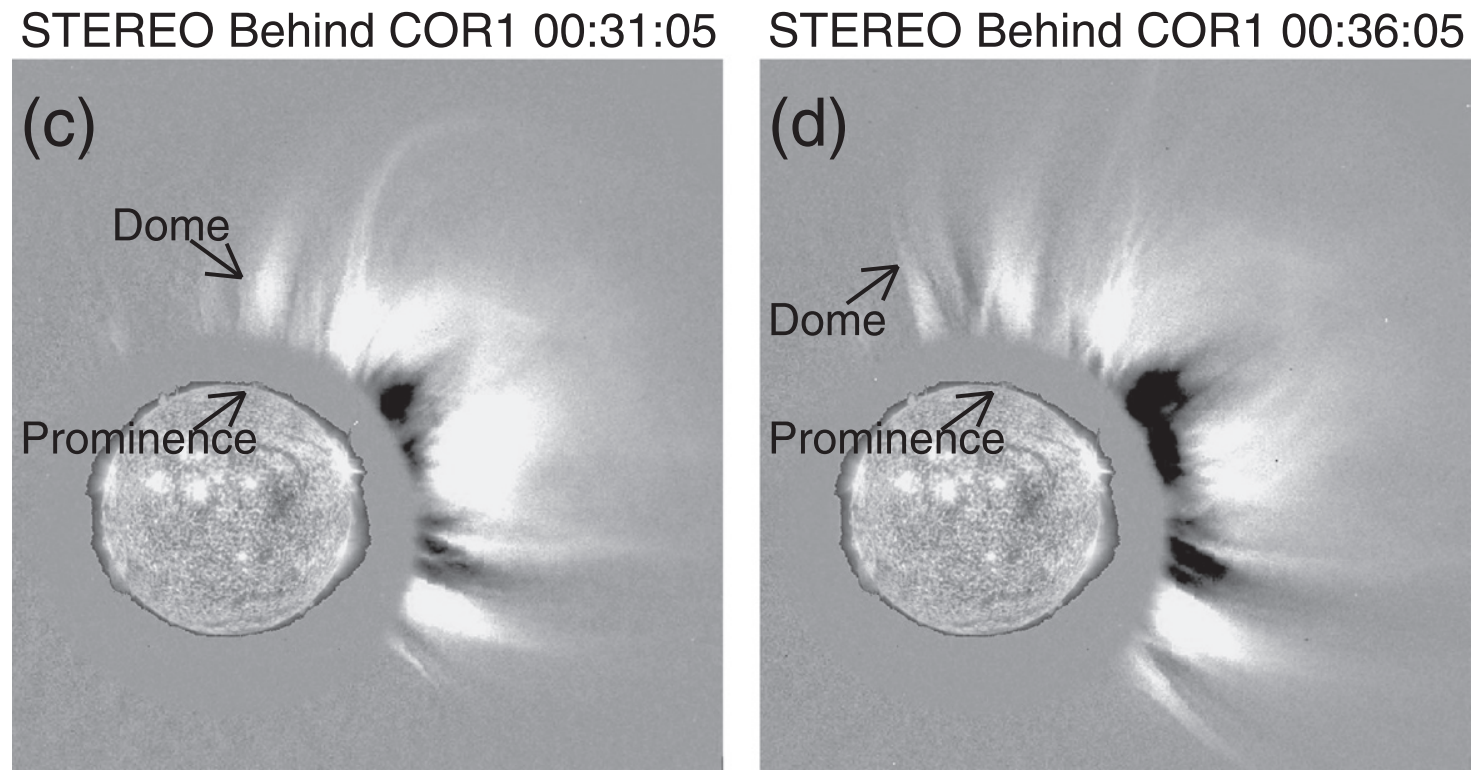

Figure 2. Side view of the Sun where we can see the polar prominence and the expanding dome-like structure. Images were taken by the STEREO Behind telescope. (a) EUVI $304 \AA$ image. We can see the polar prominence clearly. (b)-(d) Difference images of the COR1 image with the EUVI $304 \AA$ image embedded. The embedded $304 \AA$ image is the same as in (a). The disturbance ahead of the expanding CME appeared at 00:26UT, propagated to the north, and passed the sky over the polar prominence.

Magnetic field strength around the prominence could be stronger than that of ambient corona, and therefore, the fast mode shock property in the corona could change gradually while approaching the prominence. If so, especially, wave refraction due to the spatial variation of fast mode propagation velocity in the corona around the prominence affects how strongly the prominence is activated. In Figure 7, we show the time-distance plot of the intensity of AIA $193 \AA$ images along the cutting-line shown in Figure 6(a) from 00:00 UT to 02:00 UT. In Figure 7, on the other hand, we can see that the coronal fast mode shock front seen as a bright signature approaching the dark filament propagates almost in constant speed. From this, we think that the spatial variation of magnetic field strength around the activated prominence did not affect the process of prominence activation much. Although the density discontinuously changes at the corona-prominence boundary, we think it is still reasonable to assume that the magnetic field strength at the corona-prominence boundary is continuous since the length scale of overall magnetic field structure is much larger than the width of the transition layer between corona and prominence.

From the discussion above, we can express the compression ratio $r_{c}$ of the coronal shock wave using the observed propagation velocity of injected wave $C_{c}$ and the initial velocity of activated prominence $V_{t}$ as

$$
r_{c}=\frac{C_{c}}{C_{c}-V_{i}}=\frac{1}{1-(1+\sqrt{a}) \frac{V_{t}}{2 C_{c}}},
$$

where we express $\rho_{p} \rho_{c}$ as $a$. In order to simplify the problem, we treat the transmitted shock wave as a perpendicular one further on. If the local density gap between the prominence 

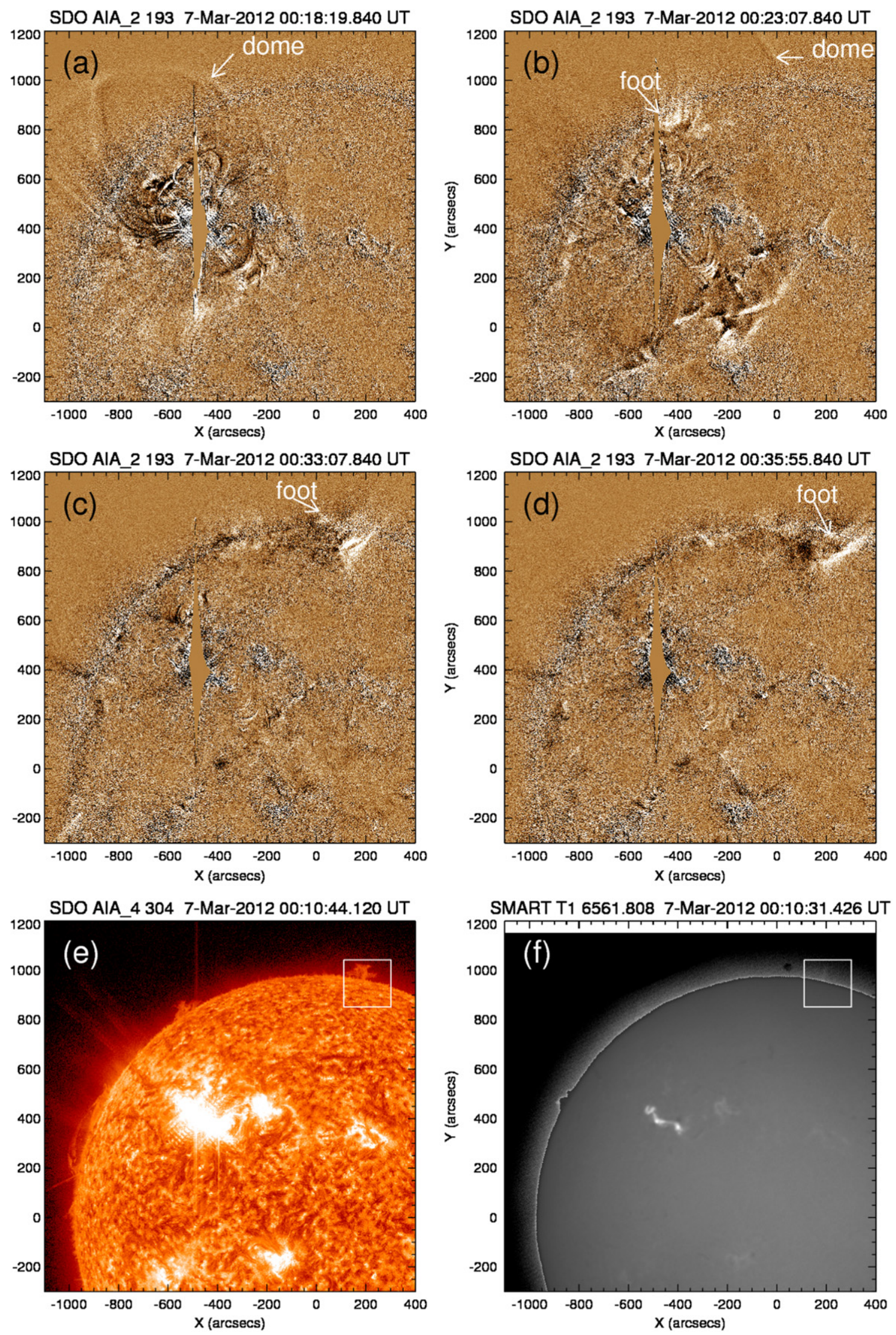

Figure 3. (a)-(d) SDO/AIA 193 Å difference image from 00:18 UT to 00:36 UT. (a) Dome-like structure appeared at 00:18 UT. (b) Footprint appeared at 00:23 UT. (c) The footprint hit the polar prominence at 00:33 UT. (d) Footprint passed the polar prominence at 00:36UT. (e) AIA 304 image at 00:10UT. We can identify the polar prominence clearly. (f) $\mathrm{H} \alpha$ image taken by SMART in Hida observatory. We can see the $\mathrm{H} \alpha$ ribbons of the flare and polar prominence. The white rectangles in (e) and (f) correspond to the FOV of Figure 5. 
(a)
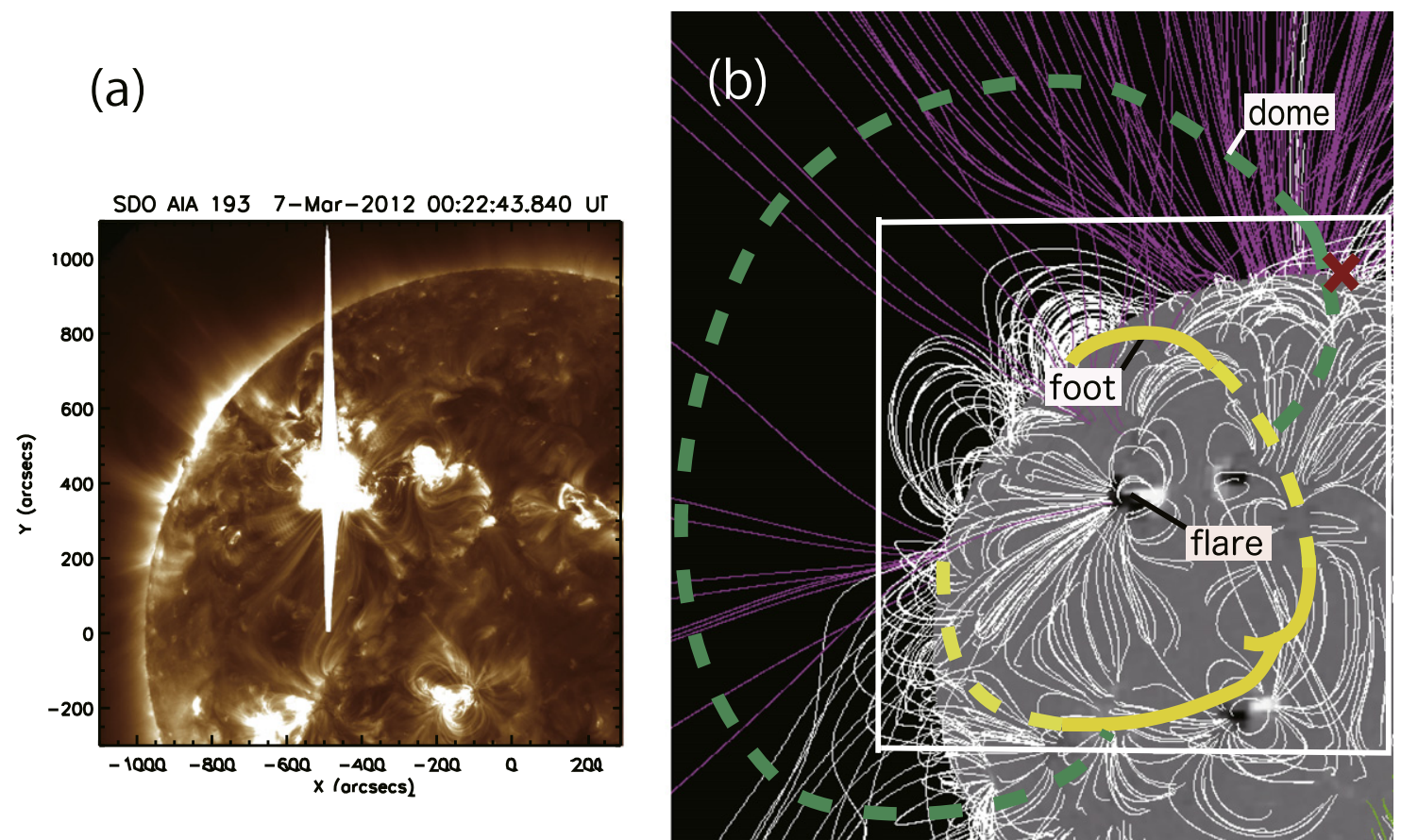

Figure 4. (a) SDO/AIA 193 AA image taken at 00:22UT. (b) Schematic figure of EUV disturbance seen in the SDO/AIA $193 \AA$ Amage (a). The line-of-sight magnetic field obtained with $S D O / \mathrm{HMI}$ before the flare and the extrapolated coronal magnetic field (potential magnetic field) is shown. The red cross expresses the location of the prominence.

and corona is $\chi$ and the volume filling factor of the prominence is $f_{V}, \rho_{p}$ can be expressed using $\rho_{c}$ as $\rho_{p}=f_{V} \chi \rho_{c}+\left(1-f_{V}\right) \rho_{c}$.

The relation between the fast mode Mach number $M_{f, c}$ and the compression ratio $r_{c}$ in perpendicular shock case is

$$
M_{f, c}=\sqrt{\frac{2 r_{c}\left((2-\gamma) r_{c}+\gamma\left(\beta_{c}+1\right)\right)}{\left((\gamma+1)-r_{c}(\gamma-1)\right)\left(\gamma \beta_{c}+2\right)}} .
$$

In the linear problem, we also relate the compression ratio $r_{p}$ in the prominence with the one in the corona $r_{c}$ as

$$
r_{p}=r_{c} \frac{2 \sqrt{a}}{1+\sqrt{a}} .
$$

We can see from Equation (6) that the compression ratio of the shock wave is enhanced when it is transmitted into a dense prominence.

From AIA $193 \AA$ images, we get $C_{c} \sin \theta=380 \mathrm{~km} \mathrm{~s}^{-1}$ and $V_{t} \sin \theta=48 \mathrm{~km} \mathrm{~s}^{-1}$ where $\theta$ is the angle between wave propagation direction and the line-of-sight direction. Here we assume the local density gap $\chi$ is 100 , the volume filling factor $f_{V}$ is between 0.001 and 0.1 (Terradas et al. 2008; Labrosse et al. 2010; Mackay et al. 2010). This leads to the range of the density gap $\rho_{p} / \rho_{c}$ between 1.1 and 10.9. Estimated coronal shock properties are shown in Table 1.

Especially, $M_{f, c}$ and $r_{p}$ fall into the range between 1.11 and 1.29, and between 1.18 and 2.11 respectively. The brightness of the prominence material in AIA $304 \AA$ images is enhanced by a factor of about two during the activation, and it is roughly consistent with the result above if the brightening is due to compression.

\section{1.5 DIMENSIONAL NUMERICAL SIMULATION OF FAST MODE WAVE TRANSMISSION}

In order to estimate the coronal shock strength, we applied the linear theory of wave transmission. In this section, we show the result of 1.5D MHD simulation of this model.

\subsection{Numerical Settings}

We numerically solved 1.5-dimensional ideal MHD equations:

$$
\begin{gathered}
\frac{\partial \rho}{\partial t}=-\frac{\partial}{\partial x}\left(\rho V_{x}\right) \\
\frac{\partial}{\partial t}\left(\rho V_{x}\right)=-\frac{\partial}{\partial x}\left(\rho V_{x}^{2}+p+\frac{1}{8 \pi} B_{y}^{2}\right) \\
\frac{\partial B_{y}}{\partial t}=-\frac{\partial}{\partial x}\left(V_{x} B_{y}\right) \\
\frac{\partial}{\partial t}\left(\frac{1}{2} \rho V_{x}^{2}+\frac{p}{\gamma-1}+\frac{1}{8 \pi} B_{y}^{2}\right) \\
=-\frac{\partial}{\partial x}\left(V_{x}\left(\frac{1}{2} \rho V_{x}^{2}+\frac{\gamma}{\gamma-1} p+\frac{1}{4 \pi} B_{y}^{2}\right)\right)
\end{gathered}
$$

The numerical scheme is the Harten-Lax-van Leer-Discontinuities approximate Riemann solver (Miyoshi \& Kusano 2005) with second-order total variation diminishing Monotonic Upstream-Centered Scheme for Conservation Laws and second order Runge-Kutta time integration with hyperbolic numerical divergence cleaning method (Dedner et al. 2002). The $x$ axis is uniformly discretized by $N=1000$ grid points. The free boundary is applied as a boundary condition and the numerical box is $x \in[-1.0,1.0]$. The ratio of specific heat $\gamma$ is assumed to be 5/3. The unit of length, 

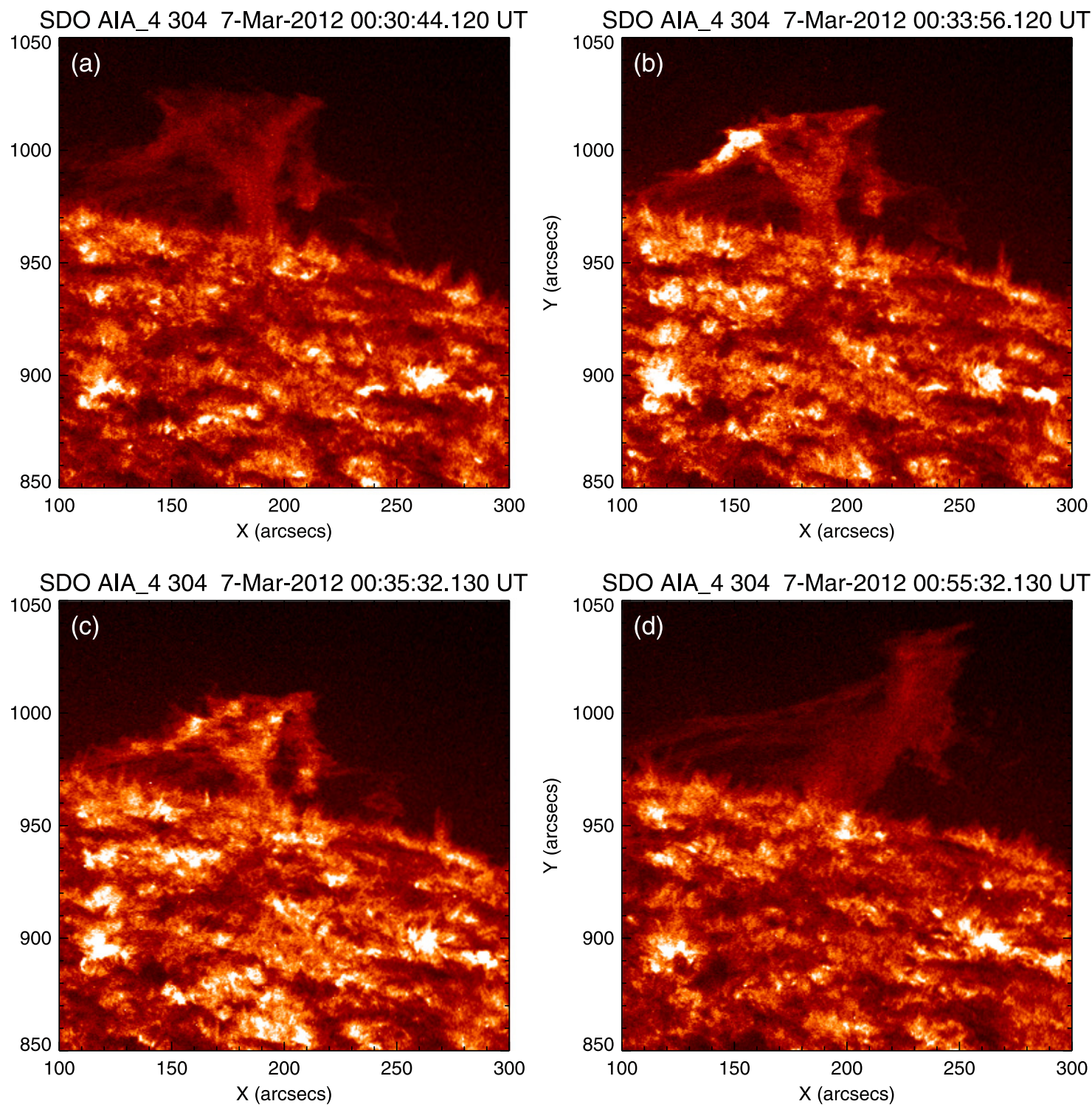

Figure 5. AIA $304 \AA$ image of prominence activation. (a) Before the arrival of the footprint at 00:31UT. (b) Just when the footprint reached the polar prominence at 00:34 UT, the edge of the prominence strongly brightened. (c) During the acceleration of the prominence by the footprint at 00:36 UT, a bright front propagates in the prominence toward the right. (d) When the prominence reached the maximum displacement at 00:55 UT after the prominence activation had completed. The prominence kept oscillating after the activation had been completed.

velocity, time, and density in the simulation are $x_{c}, V_{A c}$, $\tau=x_{c} / V_{A c}$ and $\rho_{c}$, respectively, where $V_{A c}$ is the Alfvén speed. The unit of magnetic field strength is given as $B_{c}=$ $\sqrt{\rho_{c} V_{A c}^{2}}$. The values of normalization units are set as a typical ones in the quiet Sun corona: $x_{c}=1.0 \times 10^{10} \mathrm{~cm}$, $V_{A c}=5.0 \times 10^{2} \mathrm{~km} \mathrm{~s}^{-1}, \tau=x_{c} / V_{A c}=2.0 \times 10^{2} \mathrm{~s}, \rho_{c}=1.0 \times$ $10^{-15} \mathrm{~g} \mathrm{~cm}^{-3}$ and $B_{c}=\sqrt{\rho_{c} V_{A c}^{2}}=1.6 \mathrm{G}$.

Initial conditions are as follows,

$$
\begin{gathered}
\rho= \begin{cases}\rho_{c}=1.0 & (x<0.5) \\
\rho_{p} & (0.5<x)\end{cases} \\
p=p_{0} \\
B=\left(0, B_{0}\right)
\end{gathered}
$$

$$
\boldsymbol{V}=\left\{\begin{array}{l}
\left(2 V_{0} \sin \left(\frac{2 \pi x}{w}\right), 0\right) \quad(|x|<w) \\
(0,0)(|x|>w)
\end{array}\right.
$$

where $\rho_{c}$ and $\rho_{p}$ denotes the density of the corona and prominence respectively.

Initially, gas pressure and magnetic field are set uniform. The magnetic field direction is perpendicular to the direction of wave propagation since we investigate the perpendicular shock injection problem in this study. There is a density gap at $x=0.5$, the left and right sides of which are the corona and the prominence, respectively. The density gap between the corona and the prominence is resolved by one numerical grid. The initial wave packet is the superposition of two sinusoidalshaped wave packets propagating in opposite directions of each other. We let the sinusoidal-shaped wave packet 

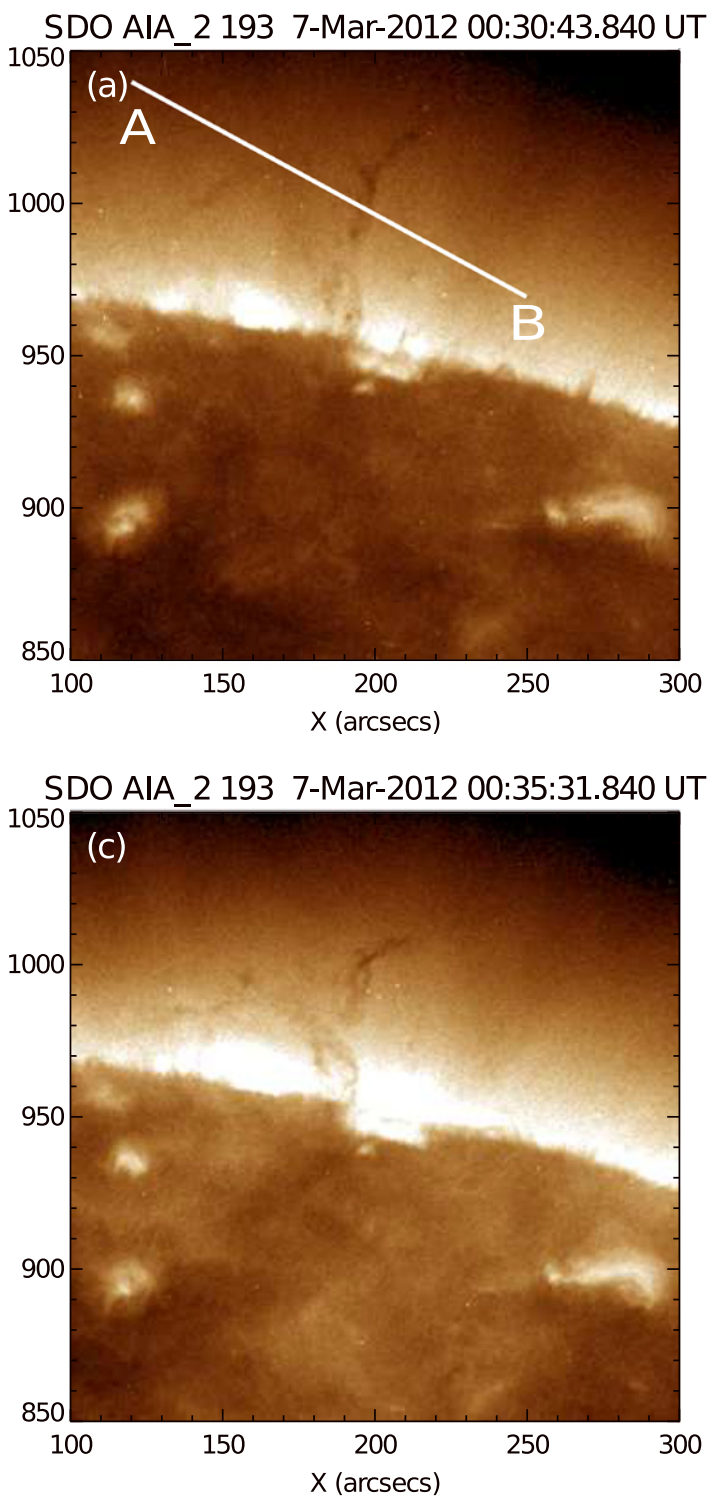

SDO AIA 2193 7-Mar-2012 00:33:55.840 UT

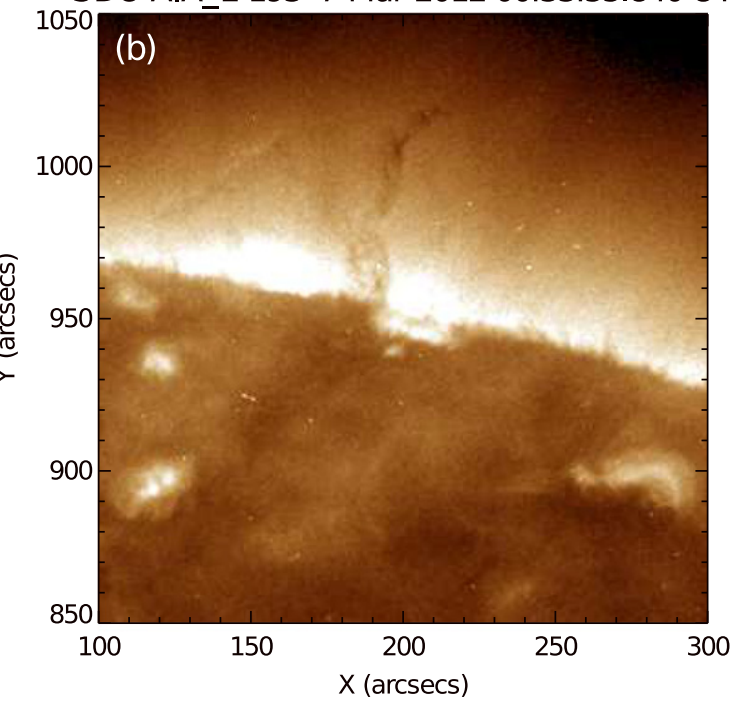

SDO AIA 2193 7-Mar-2012 00:55:31.840 UT

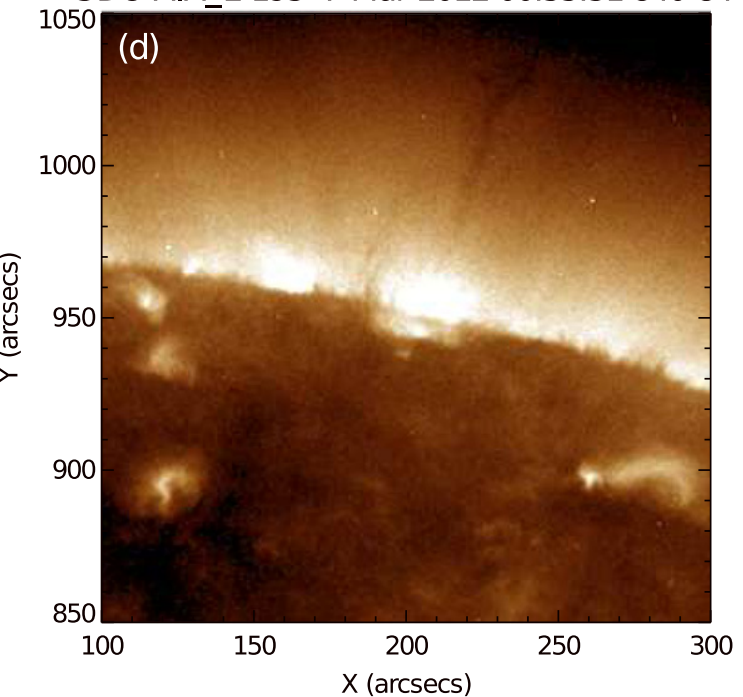

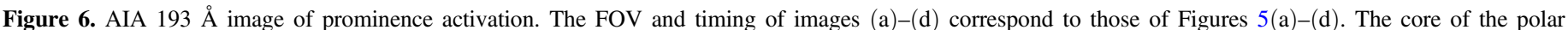

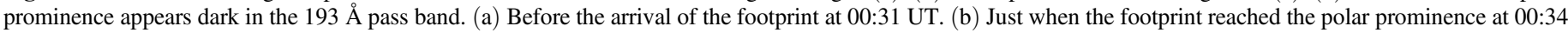

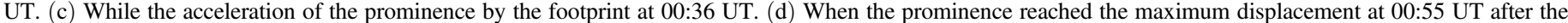
prominence activation had completed. The prominence kept oscillating after the activation had been completed.

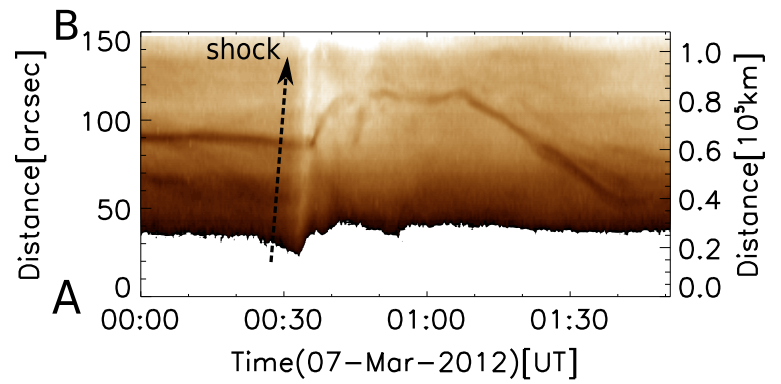

Figure 7. AIA $193 \AA$ time-distance plot of prominence activation along the line A-B in Figure 6. The pixels that have pixel counts lower than the threshold value, which is set to emphasize both the signatures of the wave front and the dark filament, are outside of the color table and appear white in the figure. The bright feature approaching the dark filament is the coronal shock front. We can see that the propagation speed of the shock front does not change much in the corona around the prominence. Just when the shock front arrives at the prominence, the prominence is suddenly accelerated. After the activation, the prominence reaches its maximum displacement and continues oscillation. propagate in the corona and go into the dense region, "the prominence."

For quasilinear cases $V_{0}=0.01$, and for nonlinear cases $V_{0}=0.2$. The width of the wave packet $w$ is set to be 0.05 . For each case, $\rho_{c}=1$, and $\rho_{p}$ ranges from 2 to 20. $B_{c}$ is fixed to be $\sqrt{4 \pi}$ so that the initial Alfvén velocity is unity in all cases. The initial pressure $p_{0}$ takes the value 0.025 or 0.1 , each of which corresponds to plasma beta $\beta=0.05$ and $\beta=0.2$. The initial distribution of $V_{x}$ and $\rho$ in quasilinear case $\left(V_{0}=0.01\right)$ with $\rho_{p}=20$ and is shown in Figure 8 . While the wave injection into the prominence, some part of the wave energy is transmitted while some parts are reflected (Figure 9). In the nonlinear case, initially the sinusoidalshaped wave packet is deformed by the nonlinear sharpening effect, and became shock before the injection into the prominence (Figure 10). 
Table 1

Estimated Shock Properties for $\gamma=5 / 3$ on the Basis of the Linear Theory

\begin{tabular}{lcccc}
\hline \hline$f_{V}{ }^{\mathrm{a}}$ & $\beta_{c}{ }^{\mathrm{b}}$ & $r_{c}{ }^{\mathrm{c}}$ & $r_{p}{ }^{\mathrm{d}}$ & $M_{f, c} \mathrm{e}$ \\
\hline 0.001 & 0.05 & 1.15 & 1.18 & 1.11 \\
0.001 & 0.20 & 1.15 & 1.18 & 1.11 \\
0.01 & 0.05 & 1.18 & 1.38 & 1.14 \\
0.01 & 0.20 & 1.18 & 1.38 & 1.13 \\
0.1 & 0.05 & 1.37 & 2.11 & 1.29 \\
0.1 & 0.20 & 1.37 & 2.11 & 1.29 \\
\hline
\end{tabular}

${ }^{a}$ The filling factor of the prominence.

b Plasma beta in the corona.

${ }^{c}$ Compression ratio of the shock wave in the corona.

${ }^{\mathrm{d}}$ Compression ratio of the shock wave in the prominence.

${ }^{\mathrm{e}}$ Fast mode Mach number of the shock wave in the corona.
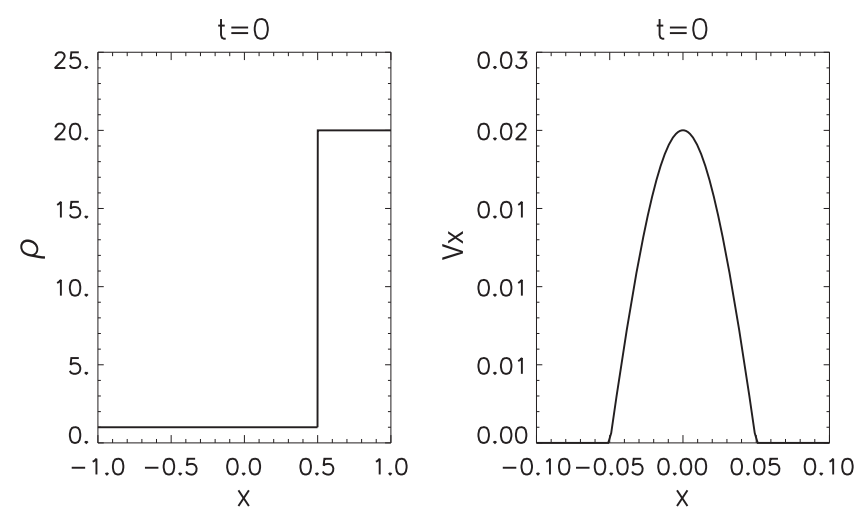

Figure 8. Initial profile of the density (left) and velocity (right) for a quasilinear case with $\rho_{p}=20$.
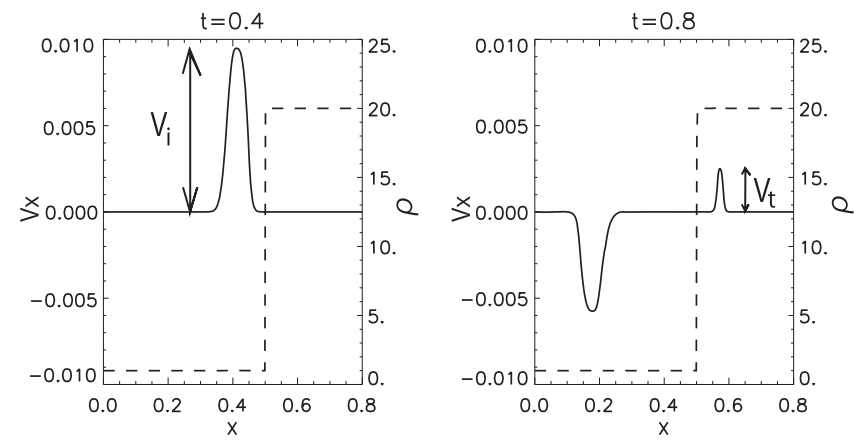

Figure 9. $V x$ just before and after the wave transmission in the quasilinear case. Velocity amplitudes of injected $\left(V_{i}\right)$ and transmitted $\left(V_{t}\right)$ waves are indicated.

In order to compare quasilinear and nonlinear cases, we define "velocity transmittance" of the wave $T_{v}$ as follows.

$$
T_{v}=\frac{V_{t}}{V_{i}}
$$

This is the ratio of the velocity amplitude of transmitted and injected waves (see Figures 9 and 10). The velocity amplitudes of injected and transmitted waves are measured just before and after the injection, respectively. Linear analytic expression of this quantity as a function of the density gap a $=\rho_{p} / \rho_{c}$ is (from
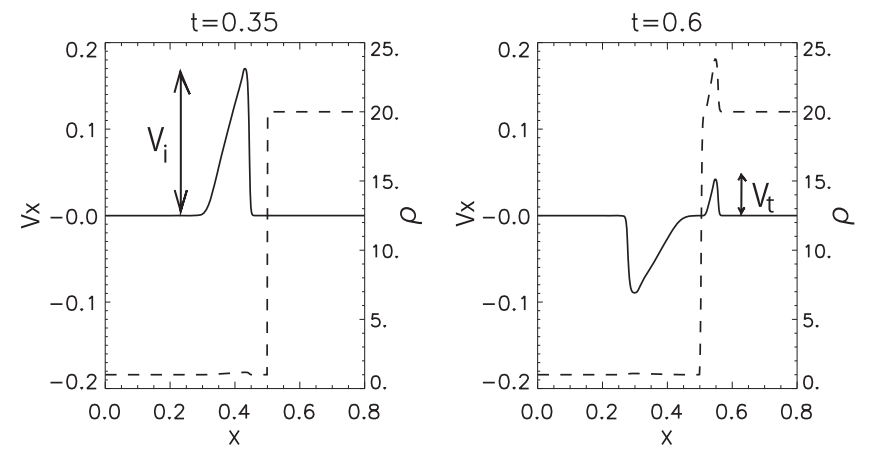

Figure 10. $V x$ just before and after the wave transmission in the nonlinear case. Velocity amplitudes of injected $\left(V_{i}\right)$ and transmitted $\left(V_{t}\right)$ waves are indicated. (a)

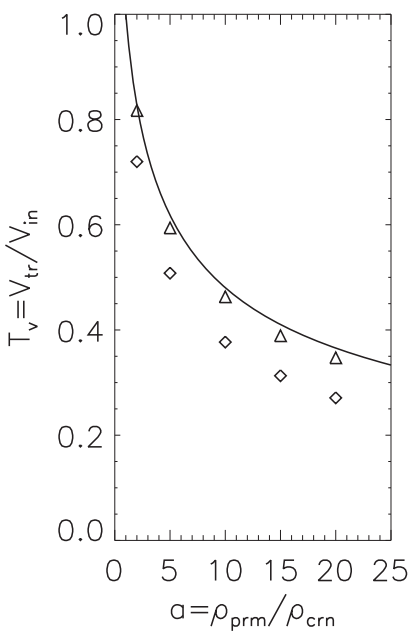

(b)

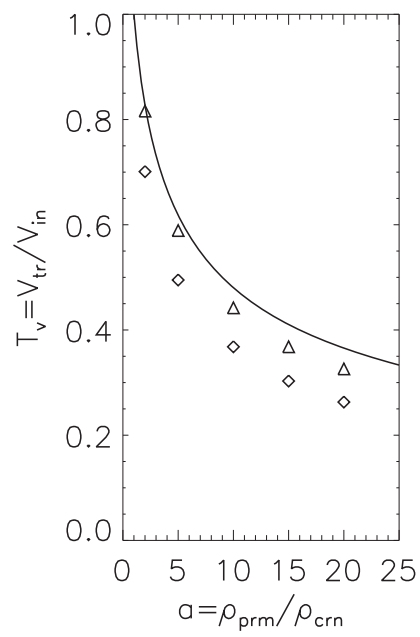

Figure 11. Result of the one-dimensional simulation of shock transmission. The velocity transmittance $T_{v}$ is shown as a function of density gap a. Solid lines denote the linear analytic solution, triangles and diamonds show the numerical results of quasilinear and nonlinear (shock) transmission cases, respectively. (a) The plasma beta $\beta=0.05$ case. Fast mode Mach number of the injected shock wave $M_{f}=1.16$. (b) The plasma beta $\beta=0.20$ case. Fast mode Mach number of the injected shock wave $M_{f}=1.17$.

Equation (3)),

$$
\mathrm{T}_{v}=\frac{2}{1+\sqrt{a}} .
$$

Figure 11 compares the numerical results and analytic expression. (a) and (b) show the cases with $\beta=0.05$ and $\beta=0.20$, respectively. From the result shown in Figure 11, we can say that if the strength of the injected shock is not strong, the linear analytic treatment is not so bad.

Then, we define value $R_{c}$ as follows

$$
R_{c}=\frac{\rho}{\rho_{0}},
$$

where $\rho_{0}$ denotes the initial density distribution. At the wave front, $R_{c}$ corresponds to the wave compression ratio. The distribution of $R_{c}$ in the quasilinear case just before and after the wave injection is shown in Figure 12, and the same plot for the nonlinear case is shown in Figure 13. The time evolution of the velocity and compression ratios in quasilinear and nonlinear 

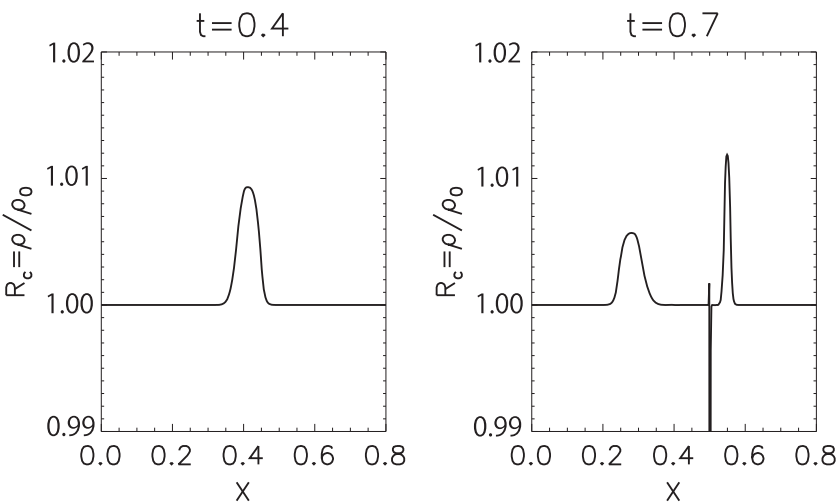

Figure 12. $R_{c}$ just before and after the wave transmission in the quasilinear case. Enhancement of $R_{c}$ after the transmission can be seen.
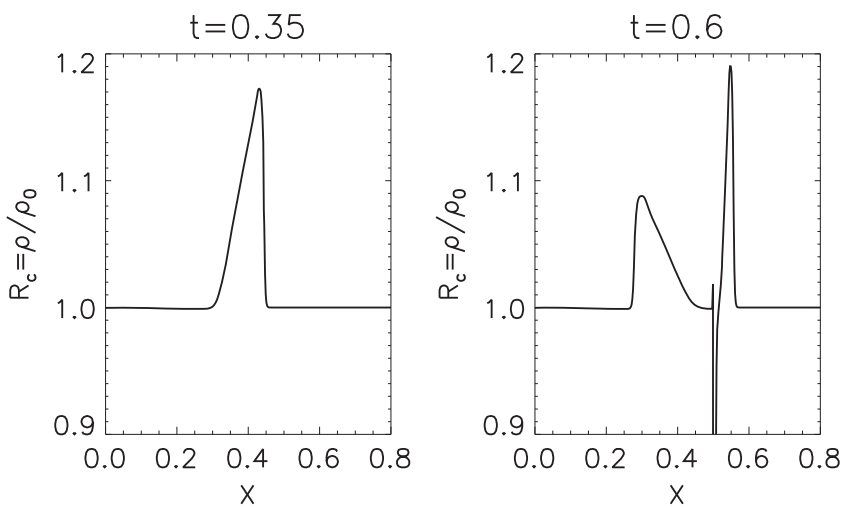

Figure 13. $R_{c}$ just before and after the wave transmission in the nonlinear case Enhancement of $R_{c}$ after the transmission can be seen.
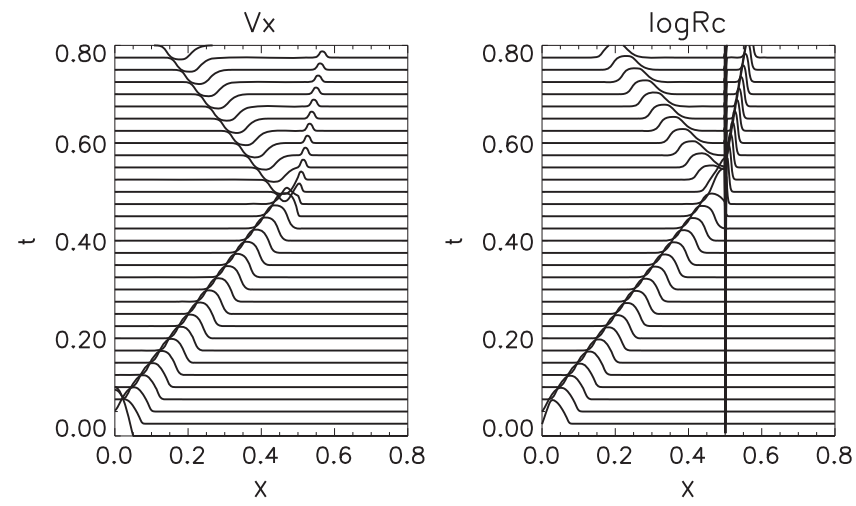

Figure 14. Time evolution of $V_{x}$ and $\log R_{c}$ in the quasilinear case is shown.
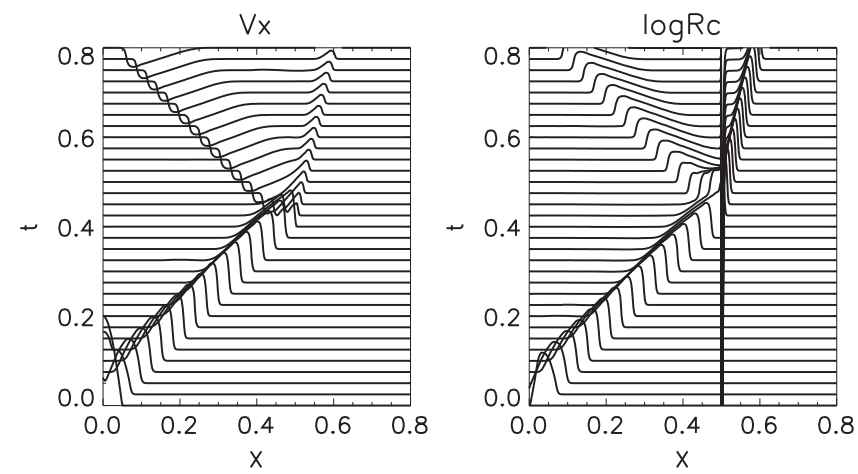

Figure 15. Time evolution of $V_{x}$ and $\log R_{c}$ in the nonlinear case is shown. cases are shown in Figures 14 and 15. The steep overshoot at $x=0.5$ after the wave injection is the result of the rightward shift of the prominence material, so it does not represent the wave compression ratio. From the linear theory, the compression ratio of the wave is expected to be enhanced after the transmission into the prominence (Equation (6)). The expected enhancement of the compression ratio after the wave transmission can be seen both in quasilinear and nonlinear cases.

\section{SUMMARY AND CONCLUSION}

In this paper we studied the nature of a globally propagated EUV wave associated with an X5.4 class flare that occurred at AR 11429 on 2012 March 7. The X5.4 class flare started at 00:02 UT and peaked at 00:24 UT. A dome-like structure was observed in the images taken by SDO/AIA at 00:18 UT and continued to expand. Then, at 00:23 UT, another disturbance front appeared in the lower corona and propagated toward the north with a velocity of around $670 \mathrm{~km} \mathrm{~s}^{-1}$. The footprint reached the polar prominence at 00:33 UT. On the other hand, in the images taken by STEREO/COR1, a disturbance appeared at 00:25 UT around the expanding CME bubble, and passed the north pole between 00:31 UT and 00:36 UT. The appearance of the disturbance agreed well with the start of the detection of the Type II radio burst by HIRAS. The time variation of the location of the EUV disturbance that propagated to the north also agreed well with the location of the disturbance seen in the STEREO/COR1 images. From these observational properties of the EUV waves, we consider the footprint to be a footprint of the shock front launched from expanding CME bubble.

When the EUV wave hit the polar prominence, it was strongly brightened and the bright part propagated in the direction of EUV wave propagation. At the same time, the prominence was accelerated toward the direction of the propagation of the wave. We consider the prominence activation to be a result of shock injection. The strong brightening of the prominence could be explained as a result of the interaction of the shock with the prominence and resulting enhancement of the compression ratio of the prominence material.

Using the observed velocity of activated prominence, the fast mode Mach number $M_{f}$ of the coronal shock wave was estimated to be between 1.20 and 1.42 on the basis of linear theory. Also, linear theory predicted the enhancement of the shock compression in the prominence material, which could explain the strong brightening of the activated prominence in AIA $304 \AA$ images. The estimated compression ratio of the shock in the prominence was between 1.74 and 2.50. We checked the validity of the method with a one-dimensional numerical model calculation and found that the linear theory is applicable when the shock is not strong.

The SDO/AIA data are courtesy of the NASA/SDOand AIA science team. The authors thank the SMART team at the Hida observatory of Kyoto University who continuously provide high quality $\mathrm{H} \alpha$ full disk images of the Sun. The simulation code used in this work was created with the help of the HPCI Strategic Program. This work is financially supported by JSPS KAKENHI grant Nos. 24740331, 23340045, and 25287039. The authors are grateful to Dr. Andrew Hillier (Kyoto University) for helpful comments. 
The Astrophysical Journal, 801:37 (11pp), 2015 March 1

\section{REFERENCES}

Asai, A., \& Ishii, T. 2011, ApJL, 745, L18

Chen, P. F., \& Wu, Y. 2011, ApJL, 732, L20

Dedner, A., Kemm, F., Kröner, D., et al. 2002, JCoPh, 175, 645

Delaboudinieře, J.-P., Artzner, G. E., Brunaud, J., et al. 1995, SoPh, 162, 291

Domingo, V., Fleck, B., \& Poland, A. I. 1995, SoPh, 162, 1

Driesman, A., Hynes, S., \& Cancro, G. 2008, SSRv, 136, 17

Eto, S., Isobe, H., Narukage, N., et al. 2002, PASJ, 54, 481

Gallagher, P. T., \& Long, D. M. 2010, SSRv, 158, 365

Gilbert, H. R., Daou, A. G., Young, D., Tripathi, D., \& Alexander, D. 2008, ApJ, 685, 629

Gopalswamy, N., Lara, A., Kaiser, M. L., \& Bougeret, J.-L. 2001, JGR, 106, 25261

Isobe, H., Tripathi, D., Asai, A., \& Jain, R. 2007, SoPh, 246, 89

Kai, K. 1970, SoPh, 11, 310

Kaiser, M. L., Kucera, T. A., Davila, J. M., et al. 2008, SSRv, 136, 5

Kondo, T., Isobe, T., Igi, S., Watari, S., \& Tokimura, M. 1995, J. Commun. Res. Lab, 42, 111

Labrosse, N., Heinzel, P., Vial, J.-C., et al. 2010, SSRv, 151, 243

Lemen, J. R., Title, A. M., Akin, D. J., et al. 2012, SoPh, 275, 17

Liu, R., Liu, C., Xu, Y., et al. 2013, ApJ, 773, 166

Liu, W., Nitta, N. V., Schrijver, C. J., Title, A. M., \& Tarbell, T. D. 2010, ApJL, 723, L53

Ma, S., Raymond, J. C., Golub, L., et al. 2011, ApJ, 738, 160

Mackay, D. H., Karpen, J. T., Ballester, J. L., Schmieder, B., \& Aulanier, G. 2010, SSRv, 151, 333

Mann, G., Jansen, F., MacDowall, R. J., Kaiser, M. L., \& Stone, R. G. 1999 , A\&A, 348, 614
Miyoshi, T., \& Kusano, K. 2005, JCoPh, 208, 315

Moreton, G. E. 1960, AJ, 65, 494

Newkirk, G. A. 1961, ApJ, 133, 983

Okamoto, T. J., Nakai, H., Keiyama, A., et al. 2004, ApJ, 608, 1124

Pesnell, W. D., Thompson, B. J., \& Chamberlin, P. C. 2012, SoPh, 275, 3

Petrie, G. J. D. 2012, ApJ, 759, 50

Shibata, K., Kitai, R., Kadoma, M., et al. 2011, Solar Activity in 1992-2003 (Kyoto: Kyoto Univ. Press)

Shibata, K., \& Magara, T. 2011, LRSP, 8, 6

Smith, S. F., \& Harvey, K. L. 1971, in Physics of the Solar Corona, ed C. J. Macris (Dordrecht: Reidel), 156

Thompson, B. J., Gurman, J. B., Neupert, W. M., et al. 1999, ApJL, 517, L151

Thompson, B. J., Reynolds, B., Aurass, H., et al. 2000, SoPh, 193, 161

Terradas, J., Arregui, I., Oliver, R., \& Ballester, J. L. 2008, ApJL, 678, L153

Title, A., \& AIA, Team 2006, BAAS, 38, 261

Tripathi, D., \& Raouafi, N.-E. 2007, A\&A, 473, 951

Tripathi, D., Isobe, H., \& Jain, R. 2009, SSRv, 149, 283

Uchida, Y. 1968, SoPh, 4, 30

Vršnak, B., Warmuth, A., Brajša, R., \& Hanslmeier, A. 2002, A\&A, 394, 299

Vršnak, B., Veronig, A. M., Thalmann, J. K., \& Žic, T. 2007, A\&A, 471, 295

Wang, S., Liu, C., \& Wang, H. 2012, ApJL, 757, L5

Warmuth, A., Vršnak, B., Aurass, H., \& Hanslmeier, A. 2001, ApJL, 560, L105

Warmuth, A. 2007, LNP, 725, 107

Wild, J. P., Murray, J. D., \& Rowe, W. C. 1954, AuJPh, 7, 439

Wills-Davey, M. J., \& Attrill, G. D. R. 2009, SSRv, 149, 325

Wülser, J.-P., Lemen, J. R., Tarbell, T. D., et al. 2004, Proc. SPIE, 5171, 111

Zhang, Y., Kitai, R., Narukage, N., et al. 2011, PASJ, 63, 685 